\title{
Self-running droplet: Emergence of regular motion from nonequilibrium noise
}

\section{$\operatorname{AUTHOR}(\mathrm{S}):$}

Sumino, Y; Magome, N; Hamada, T; Yoshikawa, K

\section{CITATION:}

Sumino, Y ...[et al]. Self-running droplet: Emergence of regular motion from nonequilibrium noise. PHYSICAL REVIEW LETTERS 2005, 94(6): 068301.

\section{ISSUE DATE:}

2005-02-18

URL:

http://hdl.handle.net/2433/49863

RIGHT:

Copyright 2005 American Physical Society 


\title{
Self-Running Droplet: Emergence of Regular Motion from Nonequilibrium Noise
}

\author{
Yutaka Sumino, Nobuyuki Magome, ${ }^{*}$ Tsutomu Hamada, and Kenichi Yoshikawa ${ }^{\dagger}$ \\ Depertment of Physics, Graduate School of Science, Kyoto University and CREST, Kyoto 606-8502, Japan
}

(Received 30 June 2004; published 14 February 2005)

\begin{abstract}
Spontaneous motion of an oil droplet driven by nonequilibrium chemical conditions is reported. It is shown that the droplet undergoes regular rhythmic motion under appropriately designed boundary conditions, whereas it exhibits random motion in an isotropic environment. This study is a novel manifestation on the direct energy transformation of chemical energy into regular spatial motion under isothermal conditions. A simple mathematical equation including noise reproduces the essential feature of the transition from irregularity into periodic regular motion. Our results will inspire the theoretical study on the mechanism of molecular motors in living matter, working under significant influence of thermal fluctuation.
\end{abstract}

DOI: $10.1103 /$ PhysRevLett.94.068301

The second law of thermodynamics prohibits specific spontaneous motion in macroscopic systems under equilibrium; nature obeys the law of equipartition. In contrast, living organisms exhibit a specific spatiotemporal structure in a self-organized manner under thermodynamically open conditions, where chemical processes play the major role. Over the past several decades, reaction-diffusion systems have attracted considerable attention as a simple model for the formation of a spatiotemporal structure in living organisms. It has been shown both experimentally and theoretically that various dissipative structures can be generated, such as traveling waves and a Turing pattern [1-3]. However, in a reaction-diffusion system there is essentially no mass flow, or geometric motion, in contrast to actual observations in living things. In the present study, we found regular spatial movement could be induced in a reactive droplet by adopting an appropriate boundary condition under chemically nonequilibrium conditions. We will discuss a novel scenario for the generation of macroscopic directed and/or periodic motion under a large fluctuation.

Since the 19th century, it has been known that an oilwater system exhibits spontaneous mechanical agitation, i.e., the Marangoni effect [4-9]. Two different kinds of self-agitation, thermal and chemical Marangoni convection, have been reported. Chemical Marangoni convection is described as the time-dependent fluctuation of interfacial tension under isothermal conditions, where the driving force is the transfer of solute through the interface. One type of chemical Marangoni convection is the spontaneous mechanical motion of a reactive liquid droplet on a solid substrate. The motion of a reactive droplet has been predicted theoretically [10-14] and verified experimentally [15-21]. However, in such a system the trajectory of motion cannot overlap itself because the substrate surface is changed in an irreversible manner, which would preclude periodic or circular motion. In the present study, we found that irregular agitation induced by a chemical Marangoni effect $[4,7,8]$ can be transduced into characteristic repetitive, rhythmic motion, simply by choosing appropriate boundary conditions.
PACS numbers: $83.80 . J \mathrm{X}, 47.70 . \mathrm{FW}$

The aqueous phase, $1 \mathrm{mM}$ stearyl trimethyl ammonium chloride (STAC), and the organic phase, $5 \mathrm{mM}$ iodine solution of nitrobenzene saturated with potassium iodide, were used. STAC was prepared through recrystallization from acetone. For glass substrates, we used microslide glass (Matsunami, Osaka; S9111) without any pretreatment. As for a measurement 20-60 $\mu 1$ of the oil was placed in an aqueous phase on a glass substrate. The movement of the oil droplet was recorded by high speed video camera (RedLake MASD, Inc. Motion Scope PCI) in 60 frames per second. All the measurements were carried out at the room temperature.

Figure 1 shows the motion of an oil droplet on a glass substrate in an aqueous phase. We put a small amount $(30 \mu \mathrm{l})$ of oil on the glass substrate through a pipette. The oil droplet spontaneously began to move immediately after it was transferred to the glass substrate. As shown in Fig. 1(a), the oil droplet exhibited random agitation within the circular glass vessel. Note that the trajectory of the oildroplet motion crosses itself. In contrast to the irregular motion shown in Fig. 1(a), a regular rhythmic motion can be generated in the same chemical system simply by changing the spatial geometry, as in Fig. 1(b). Corresponding movies are available online [22]. Such periodic motion along the glass substrate continues over several tens of seconds and then stops. Figure 1 also shows time traces of the $x$ component of velocity, together with their Fourier transformation, indicating the appearance of periodicity $f \approx 0.5 \mathrm{~Hz}$ for motion on the glass stripe (b) in contrast to the noisy behavior in the circular vessel (a). Careful observation of Fig. 1(b) reveals that the advancing contact angle of the oil droplet was smaller than its receding contact angle. Figure 1(c) shows the enlarged image of an oil droplet moving towards the right, where the advancing contact angle is greater than its receding angle. This large difference in contact angles suggests that the driving force can be attributed to the difference in surface tension between the front and the rear of the oil droplet.

Next, we conducted an experiment to check for differences in the surface of the glass plate by mimicking the 
(a)
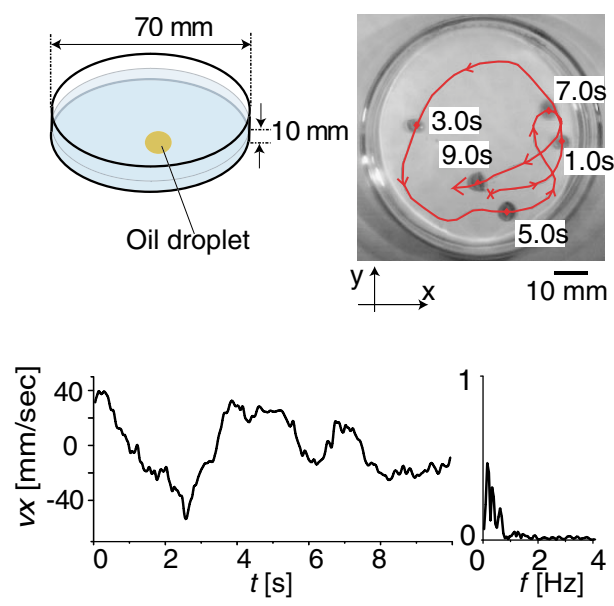

(b)
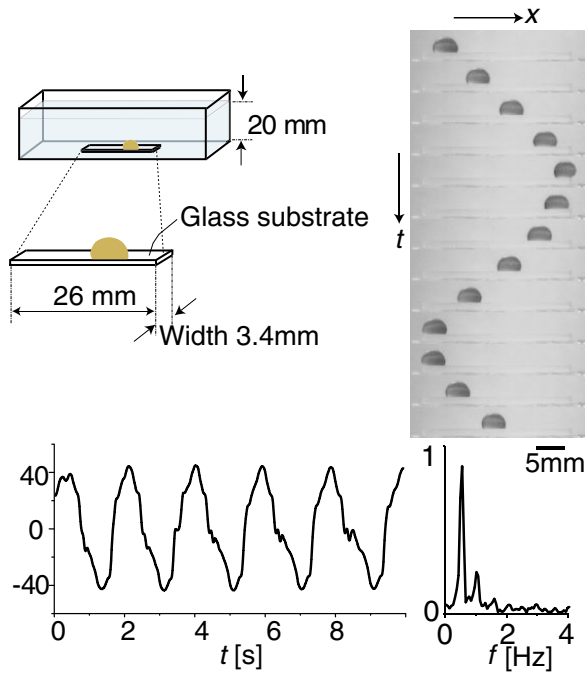

(c)
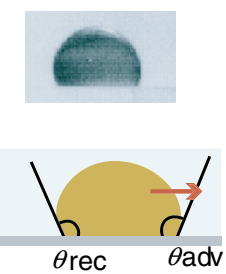

FIG. 1 (color). According to the configuration of the glass substrate, an oil droplet showed a distinct mode of motion. (a) Upper left: Schematic diagram of the experimental setup. The volume of the oil droplet is $30 \mu 1$. Upper right: Random motion appears under isotropic conditions. Also see movies in [22]. Lower panel: Trace of the $x$ component of oil-droplet velocity and its Fourier transformation, where there is no distinct peak. The $y$-component velocity shows similar behavior. (b) Upper left: Schematic diagram of the experimental setup. The volume of the oil droplet is $30 \mu l$. Upper right: Periodic forward-and-backward motion appears on a narrow and straight glass substrate. The time scale in the picture on the right is $1 / 6 \mathrm{~s}$ per frame. The rhythmic motion continues for $10 \mathrm{~s}$. Also see movies in [22]. Lower panel: Trace of the $x$ component of oil-droplet velocity and its Fourier transformation, indicating a peak at $0.5[\mathrm{~Hz}]$. (c) Schematic representation of an oil droplet, moving toward the red arrow. $\theta_{\text {adv }}$ was smaller than $\theta_{\text {rec }}$. On average, $\theta_{\mathrm{adv}} \approx 105^{\circ}, \theta_{\mathrm{rec}} \approx 116^{\circ}$.

conditions before and after the oil droplet had passed. We examined the contact angle of a droplet with pure water on the glass plates A and B under air. The glass plate A was pretreated with the procedure of immersion in an aqueous solution for $5 \mathrm{~min}$, rinsed with distilled water, and then dried in the air. The other substrate B was pretreated in the same procedure as plate $A$, then dipped into an organic phase for $5 \mathrm{~min}$, carefully rinsed with distilled water, and dried in the air. A large difference in the contact angle was observed $\left[\theta_{\mathrm{A}} \approx 68^{\circ}, \theta_{\mathrm{B}} \approx 43^{\circ}\right.$ ] [Fig. 2(a)], indicating that plate $\mathrm{B}$ is less hydrophobic than plate $\mathrm{A}$. Thus, it is apparent that a difference in surface tension $\Delta \gamma$ between the front and the rear of an oil droplet is generated accompanied by its translational motion. Once $\Delta \gamma$ becomes nonzero due to some spontaneous small motion driven by the Marangoni effect, an oil droplet tends to continue the directed motion by avoiding the backward movement due to the memory effect of the chemical condition $[14,19]$.

The generation of a difference in surface tension between the front and the rear can be explained as schematically shown in Fig. 2(b). Since the surface of the glass substrate has a negative charge, $\mathrm{STA}^{+}$(Stearyl Trimethyl Ammonium Ion) is arranged on the glass plate with the head group attached to the surface and the hydrophobic tail toward the aqueous phase; as a result, the glass surface that faces the aqueous solution is hydrophobic. When an oil droplet exhibits a directed motion due to the Marangoni effect, $\mathrm{STA}^{+}$attached to the surface tends to dissolve into the organic phase. Consequently, the glass surface becomes less hydrophobic, and the difference in surface tension is generated between the front and the rear of the droplet. This imbalance in the surface tension promotes directed motion against viscous damping. The existence of $\mathrm{I}_{3}^{-}$made from $\mathrm{I}^{-}$and $\mathrm{I}_{2}$ in an oil droplet accelerates the transfer of $\mathrm{STA}^{+}$from the surface into the organic phase [8] by creating a hydrophobic ion pair with $\mathrm{STA}^{+}$. Significantly, the surface of the glass substrate spontaneously returns to the hydrophobic state soon after an oil droplet passes by, since $\mathrm{STA}^{+}$in the aqueous phase tends to be absorbed onto the glass substrate. This mechanism allows the trajectory of an oil droplet to cross itself; thus, rhythmic and repetitive motion is generated in our system.

An oil droplet ceases its motion accompanied with the decrease of the nonequilibrium chemical conditions. This is explained as follows. As $\mathrm{I}_{3}^{-}$concentration decreases in the oil droplet because of pairing with $\mathrm{STA}^{+}$, the oil droplet cannot afford enough driving force, then the oil droplet stops. We have also found that with the decrease of the size of droplet, its translational speed tends to be larger and the lifetime of self-motion to be shorter. This may be due to the enhanced surface effect on smaller systems.

Let us now discuss the mechanism of the appearance of regular, rhythmic motion in the self-agitating system. The characteristic feature of the motion of an oil droplet can be described as

$$
m \frac{d \boldsymbol{v}}{d t}=-\eta \boldsymbol{v}+\epsilon \frac{\boldsymbol{v}}{|\boldsymbol{v}|}+\boldsymbol{\xi}(t)
$$

The first term represents the resistance force and the sec- 
(a)

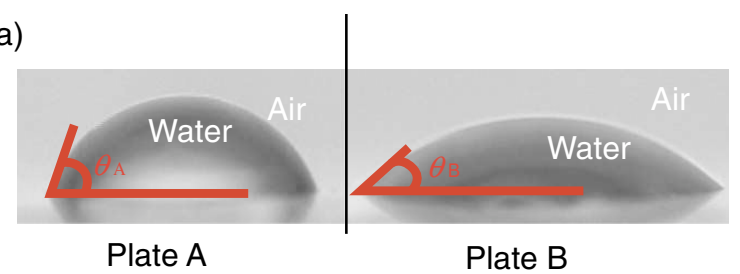

(b)
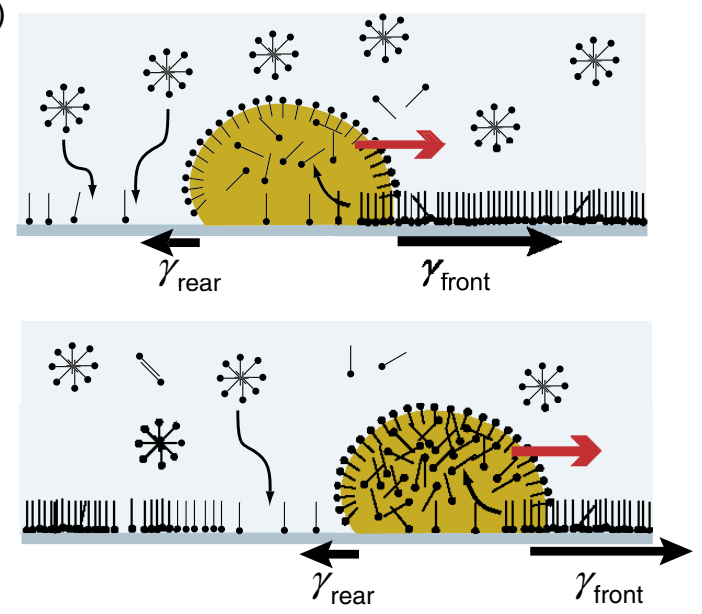

FIG. 2 (color). (a) A water droplet on a glass substrate in air. We estimate the difference in water-glass surface tension $\Delta \gamma$ from $\Delta \gamma=\gamma_{\text {pure water }}\left(\cos \theta_{\mathrm{B}}-\cos \theta_{\mathrm{A}}\right)$. Since $\theta_{\mathrm{A}} \approx 68^{\circ}, \theta_{\mathrm{B}} \approx$ $43^{\circ}$, and $\gamma_{\text {pure water }} \approx 72[\mathrm{mN} / \mathrm{m}]$, the difference in the interfacial tension is $\Delta \gamma \approx 14[\mathrm{mN} / \mathrm{m}]$. (b) Schematic diagram of the mechanism of the difference in surface tension. Red arrows show the direction of oil-droplet motion. $\mathrm{STA}^{+}$is represented as a hydrophobic bar with a polar head. Since $\mathrm{STA}^{+}$tends to assemble with $\mathrm{I}_{3}^{-}$in the droplet, $\mathrm{STA}^{+}$dissolves into the oil droplet from the glass surface. Thus, a difference surface tension is generated as $\gamma_{\text {front }}>\gamma_{\text {rear }}$. The glass surface returns to hydrophobic shortly after an oil droplet passes by, since $\mathrm{STA}^{+}$in the aqueous phase again adheres to the glass surface.

ond term represents the driving force caused by the difference in surface tension, where we incorporate the characteristics of self-motion to prohibit backward motion as in Fig. 2(b). The third term $\boldsymbol{\xi}$ represents the Gaussian random force whose standard deviation is $\sigma$ resulting from the inhomogeneity of the glass surface and the oil-water interface. Figure 3 shows schematic examples of simulated oildroplet motion based on Eq. (1) [23]. We consider that the driving force is comparable to the Gaussian random force $(\epsilon \sim \sigma)$. As for the boundary condition corresponding to the edge of a glass substrate, an oil droplet is assumed to exhibit inelastic rebound at the boundary since there exists dissipation caused by inversing convective flow inside the oil droplet. In the absence of edge effect on a vessel, random motion is generated [Fig. 3(a)]. In contrast, when the width of the glass substrate $d$ becomes narrower, i.e., when a quasi-one-dimensional boundary condition is imposed, periodic regular motion is generated [Fig. 3(b)] [24]. This result indicates that the reduction of the effective freedom of motion with an appropriate boundary condition can cause a transition from irregular behavior to regular behavior for such a droplet under nonequilibrium chemical conditions.

An oil droplet shows various types of regular motion depending on the shape of the glass substrate. Figure 4(a) shows an oil droplet moving around a vertical circle, like a roller coaster [25]. The droplet can move up the circle against gravity, where the density of the oil droplet $\rho_{\text {oil }} \approx$ 1.2 and the density of the aqueous phase $\rho_{\text {water }} \approx 1.0$. Figure 4(b) shows the stepping-up motion of an oil droplet. Based on observations in multiple experiments, we have found that the oil droplet tends to climb up the stairs rather than to fall down them. Corresponding movies are available online [22].

In the present study, we have realized regular motion under nonequilibrium noise by setting suitable boundary conditions, where the broken spatial symmetry in the container of the oil-water system reduces the freedom of motion, inducing regular motion of the droplet. Recent studies on single motor proteins [26] have indicated that the mode of such mechanical motion can be switched by changing the internal and boundary conditions [27-29]. Although the spatial scale of molecular motors in living organisms is much smaller than that of the present experimental system, the generation of regular motion under nonequilibrium in a real experiment may inspire the theoretical consideration of the working mechanism of biological molecular motors.

This work is supported by the Grant-in-Aid for the 21st Century COE "Center for Diversity and Universality in Physics" from the Ministry of Education, Culture, Sports, Science and Technology (MEXT) of Japan and by the Japan Space Forum. (a)

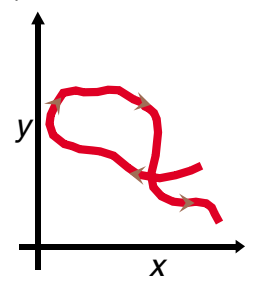

(b)

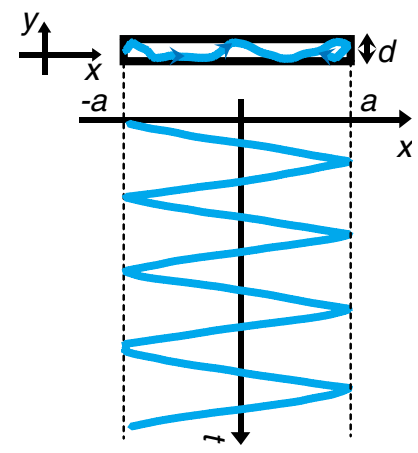

FIG. 3 (color). Numerical simulation on the spontaneous motion of an oil droplet at different boundary condition [23]. (a) Random motion of an oil droplet under boundary-free isotropic environment, which is shown as the two-dimensional trace of the motion. (b) Repetitive motion of an oil droplet on a narrow stripe that restricts the motion to $-a<x<a$ and $-d / 2<y<$ $d / 2$, where the upper panel shows the trace of the motion in real space and the lower panel shows spatiotemporal representation of the motion. Oil-droplet motion described by Eq. (1) varies distinctively depending on the effective freedom of motion that is restricted by a boundary condition. In (b), an oil droplet shows forward and backward motion when $d$ is small enough. 


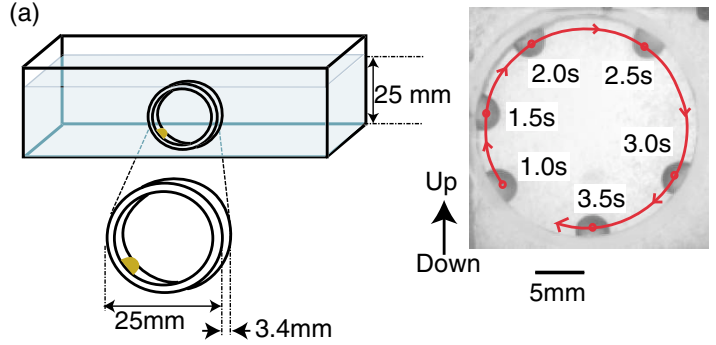

(b)
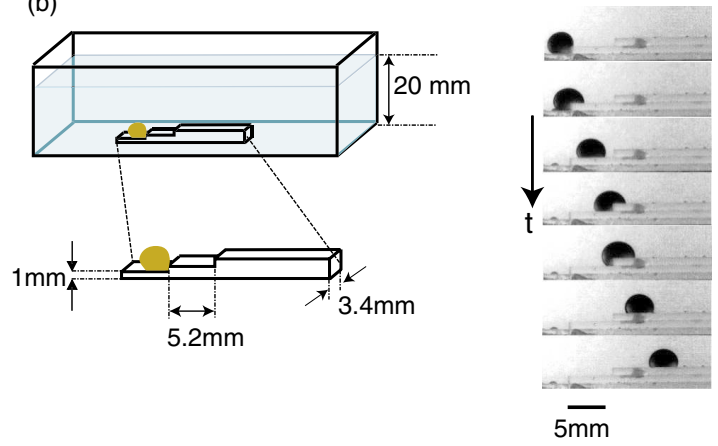

FIG. 4 (color). (a) Rotational motion within a circle normal to the horizontal plane. In the experiment shown in this figure, the droplet underwent rotation up to 3 times and then stops. The volume of the oil droplet is $20 \mu \mathrm{l}$. Also see movies in [22]. (b) Climbing motion on a stairlike substrate. The oil droplet tends to move up the stairs, implying that the stairlike substrate can regulate the direction of motion. The time interval in the pictures on the right is $1 / 6 \mathrm{~s}$ per frame. The volume of the oil droplet is $60 \mu$ l. Also see movies in [22].

*Present address: Department of Food and Nutrition, Nagoya Bunri College, Nagoya 451-0077, Japan.

${ }^{\dagger}$ To whom correspondence should be addressed. Fax: 011-81-75-753-3779

Electronic address: yoshikaw@ @scphys.kyoto-u.ac.jp

[1] G. Nicolis and I. Prigogine, Self Orgainization in Nonequilibrium Systems (Wiley, New York, 1977).

[2] J.D. Murray, Mathematical Biology (Springer-Verlag, Berlin, 1990).

[3] H. Mori and Y. Kuramoto, Dissipative Structures and Chaos (Springer-Verlag, Berlin, 1997).

[4] M. Dupeyrat and E. Nakache, Bioelectrochem. Bioenerg. 5, 134 (1978).

[5] T. Takahashi, H. Yui, and T. Sawada, J. Phys. Chem. B 106, 2314 (2002).

[6] N. M. Kovalchuk and D. Vollhardt, Phys. Rev. E 69, 016307 (2004).

[7] N. Magome and K. Yoshikawa, J. Phys. Chem. 100, 19102 (1996).

[8] A. Shioi, K. Katano, and Y. Onodera, J. Colloid Interface Sci. 266, 415 (2003).

[9] K. Yoshikawa, T. Omochi, Y. Matsubara, and H. Kourai, Biophys. Chem. 24, 111 (1986).

[10] H. P. Greenspan, J. Fluid Mech. 84, 125 (1978).

[11] P. G. de Gennes, Rev. Mod. Phys. 57, 827 (1985).

[12] F. Brochard, Langmuir 5, 432 (1989).

[13] P. G. de Gennes, F. Brochard, and D. Quéré, Capillarity and Wetting Phenomena Drops, Bubbles, Pearls, Waves (Springer-Verlag, Berlin, 2004).
[14] P. G. de Gennes, Physica (Amsterdam) 249A, 196 (1998).

[15] C.D. Bain and G. M. Whitesides, Langmuir 5, 1370 (1989).

[16] M. K. Chaudhury and G. M. Whitesides, Science 256, 1539 (1992).

[17] C.D. Bain, G. D. Burnett-hall, and R. R. Montgomerie, Nature (London) 372, 414 (1994).

[18] F. D. D. Santos and T. Ondarçuhu, Phys. Rev. Lett. 75, 2972 (1995).

[19] S. Lee and P.E. Laibinis, J. Am. Chem. Soc. 122, 5395 (2000).

[20] J. Bico and D. Quéré, Europhys. Lett. 51, 546 (2000).

[21] S. W. Lee, D. Y. Kwok, and P. E. Laibinis, Phys. Rev. E 65, 051602 (2002).

[22] See EPAPS Document No. E-PRLTAO-94-066507 for movies of irregular motion (movie1.mpg), shuttling motion (movie2.mpg), rotating motion (movie3.mpg), and climbing motion on stairlike substrates (movie4.mpg). Every movie shown is in half speed of its real motion. Detailed setup is explained in suminomovietext.doc, which is also in EPAPS Document. A direct link to this document may be found in the online article's HTML reference section. The document may also be reached via the EPAPS homepage (http://www.aip.org/ pubserves/epaps.html) or from ftp.aip.org in the directory /epaps/. See the EPAPS homepage for more information.

[23] Simulation was performed through the fourthorder Runge-Kutta method with the time step of 0.0001 . Each parameter was taken to be as follows: $\eta / m=24$, $\epsilon / m=0.9, \quad \sigma / m=10, \quad d=0.001, \quad a=0.013, \quad$ and (coefficient of rebound) $=0.5$.

[24] The occurrence of the quasi-one-dimensional motion in the simulation is understood as follows. From Eq. (1), the characteristic velocity $v_{c}$ and the relaxation time $\tau_{r}$ are given as $v_{c}=\epsilon / \eta$ and $\tau_{r}=m / \eta$. The characteristic time span $\tau_{c}$ between each rebound is $\tau_{c}=d / v_{c}=\eta d / \epsilon$, where $d$ is the free width of glass substrate. We have concluded that, when $\tau_{c} \ll \tau_{r}$, i.e., when $d$ is small enough, the velocity that is perpendicular to long axis of substrate is suppressed as long as there is loss of velocity at each rebound.

[25] We can estimate the difference in surface tension from the result of Fig. 4(a), where an oil droplet moves up vertically on a glass substrate. In this case, the driving force generated by the difference in surface tension, $\Delta \gamma$, between the rear and front should be greater than the gravitational force, $f_{\text {grav }}$, acting on the oil droplet. For the experiment in Fig. 4(a), $w \Delta \gamma=f_{\text {grav }} \geq 0.04[\mathrm{mN}]$, where $w$ is the width of the oil droplet. Since $w \approx 3.4[\mathrm{~mm}], \Delta \gamma$ should be larger than $11[\mathrm{mN} / \mathrm{m}]$, which is the same order as the acquired value in Fig. 2(a).

[26] M. Schliwa and G. Woehlke, Nature (London) 422, 759 (2003).

[27] S. A. Endow and H. Higuchi, Nature (London) 406, 913 (2000).

[28] Y. Okuda and N. Hirokawa, Proc. Natl. Acad. Sci. U.S.A. 97, 640 (2000).

[29] Y. Inoue, A. H. Iwane, T. Miyai, E. Muto, and T. Yanagida, Biophys. J. 81, 2838 (2001). 